\title{
AGS Pulsed Quadrupoles: History and Future ${ }^{1}$
}

\author{
A. D. Krisch \\ Spin Physics Center, University of Michigan, Ann Arbor, MI 48109-1120 USA
}

\begin{abstract}
This paper contains a brief discussion of the AGS polarized beam work in the 1980's. It also suggests that it might be wise to again use the old pulsed quadrupoles to overcome the problematic weak intrinsic depolarizing resonances.
\end{abstract}

Leif Ahrens earlier presented a very nice talk on an analysis of the results from the AGS polarized proton beam runs of the 1980s. I would like to now discuss some of the hardware that was used in these runs, with the goal of seeing if any of it could be used to help increase the AGS polarization in this Millennium. Using some existing hardware has many advantages, especially when a run is approaching. A discussion of this AGS polarized beam hardware can be found in great detail in a long Physical Review paper [1], published in 1989, and based on F. Z. Khiari's PhD Thesis. I will not reproduce here the many figures of the hardware or the data from their use, since they can be found in this paper.

Instead I will focus on the possible future use of one hardware item: some of the twelve pulsed quadrupoles. These 1.6- $\mu$ s-rise time ferrite quadrupoles and their 20 MW power supplies were certainly the most difficult and expensive part of the $\$ 10$ Million AGS Polarized Proton Beam Project. Moreover, they did not do a completely adequate job of overcoming the strong intrinsic depolarizing resonances at the AGS; however, they were rather successful in overcoming the weak intrinsic polarizing resonances [1]. I stress this point because the new rf dipole technique, developed in the past few years by this Millennium's AGS polarized beam team [2], has been fairly successful at overcoming the AGS's strong intrinsic depolarizing resonances, but less successful at overcoming its weak intrinsic depolarizing resonances. Thus, perhaps one should now consider:

- using the old pulsed quadrupoles to overcome the weak intrinsic depolarizing resonances;

- using the new rf dipole technique only for the strong intrinsic depolarizing resonances, where it seems to work rather well.

The new technique uses an $\mathrm{rf}$ dipole, whose frequency is moved very close to a strong intrinsic depolarizing resonance. This proximity enhances the strength of the

\footnotetext{
${ }^{1}$ Supported by a Research Grant from the U.S. Department of Energy
} 
strong intrinsic depolarizing resonance enough to allow it to spin-flip the beam almost totally, thus maintaining almost all the beam polarization. If the intrinsic depolarizing resonance is fairly strong, then one does not have to move the rf frequency too close to it; thus the beam is not destroyed. However, to use this technique for a weak intrinsic depolarizing resonance, one must move the $\mathrm{rf}$ frequency very close to the weak intrinsic depolarizing resonance; then, it becomes difficult to avoid a beam blow-up resonance, which can destroy all or part of the beam. Thus for the weak intrinsic depolarizing resonances, one is caught between two competing goals:

- maintaining the beam polarization;

- maintaining the beam intensity.

Even with perfect AGS betatron tune stability, this technique may be difficult for routine operation.

Since this new technique's problem is most serious for the weak intrinsic depolarizing resonances, and since the still-existing 1.6- $\mu$ s-rise time ferrite quadrupoles worked rather well for them [1], it seems wise to consider using these quadrupoles for this part of the job. I think that I first discussed this idea with Mei Bai and Andreas Lehrach during the SPIN 2002 Excursion to the Museum of Natural History in Manhattan; this possibly valuable discussion might be mentioned the next time anyone objects to excursions at Symposia.

The main problem with using the pulsed quadrupoles, is that their $22 \mathrm{MW}$ power supplies are in even worse shape than they were in the 1980s, when they caused many problems for many people, especially Larry Ratner and me. Fortunately, one can overcome the weak intrinsic depolarizing resonances with a fairly small betatron tune jump and thus a fairly slow rise time. Therefore, some of the old $22 \mathrm{MW}$ power supplies might work well enough to operate at a few MW; or, if they are completely dead, it might not cost too much to replace them with some much lower power devices.

Therefore, I prepared Table 1, which demonstrates several different possible plans for using three different techniques for overcoming the three main polarization problems [3] in the AGS:

1. many imperfection depolarizing resonances;

2. some weak intrinsic depolarizing resonances;

3. some strong intrinsic depolarizing resonances.

In a way it is sad that one cannot find a way to find a single elegant device, such as the Siberian snake, to overcome all depolarization problems at the AGS. However, even Yaroslav Derbenev, who is attending this Workshop cannot always produce miracles on demand. Unfortunately, the AGS is at just the wrong energy for a full Siberian snake:

- its injection energy is too low for a practical helical or dipole snake to fit in its straight sections;

- its maximum energy is too high for a solenoid snake to fit in its straight sections.

Thus, in the absence of any new miracle, one should seriously consider, the less elegant, but hopefully practical solution of using three techniques for three problems. 
TABLE 1. Overcoming the AGS Depolarizing Resonances

HISTORY

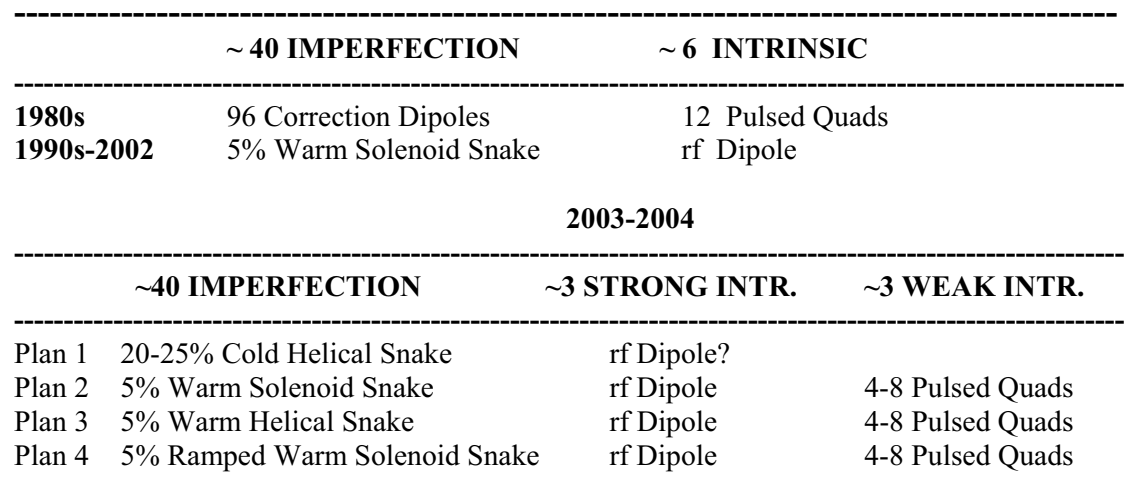

\section{REFERENCES}

1. Khiari, F.Z. et al., Phys. Rev. D39, 45-85 (1989).

2. Roser, T. et al., First talk in this Workshop and references therein.

3. Note added in proof: There may also be some coupling resonances, which Thomas Roser considered in choosing the schemes described in the Summary Talk. 